Ann. Biol. anim. Bioch. Biophys., I974, 14 (I), I45-I56.

\title{
INFLUENCE DU CALCIUM ALIMENTAIRE SUR LES CALCIFICATIONS RÉNALES, LA MAGNÉSÉMIE ET LE MAGNÉSIUM OSSEUX DU RAT CARENCÉ EN MAGNÉSIUM
}

\author{
Y. RAYSSIGUIER et P. LARVOR \\ avec la collaboration technique de M. GENEST \\ Station de Physiopathologie de la Nutrition. \\ Centre de Recherches zootechniques et vétérinaires, I. N.R. A., \\ Theix, 63110 Beaumont

\section{RÉSUMÉ}

L'évolution de la carence en magnésium est étudiée chez le Rat nourri avec des régimes à différentes teneurs en calcium. L'administration d'un régime riche en calcium aggrave le déficit magnésique mais inhibe les calcifications rénales qui accompagnent classiquement la carence en magnésium (essai no $\mathrm{I}$ : résultat obtenu après 2 semaines de carence).

Au début de la carence en magnésium des calcifications rénales apparaissent quelle que soit la teneur du régime en calcium mais disparaissent après 8 jours d'un régime riche en calcium (essai $\left.n^{\circ} 2\right)$

La distribution d'une ration enrichie en calcium pendant I semaine avant le début de la carence en magnésium ne permet pas d'inhiber les calcifications rénales précoces plus efficacement que la surcharge calcique donnée seulement en même temps que la ration carencée en magnésium (essai no 3 ).

Plusieurs hypothèses sont envisagées pour expliquer l'inhibition de la néphrocalcinose par une surcharge en calcium (activité parathyroïdienne, sécrétion de calcitonine, modification de l'élimination urinaire de calcium et phosphore).

La carence en magnésium s'accompagne de calcifications au niveau rénal et cardiovasculaire chez de nombreuses espèces comme le Rat, le Veau, le Chien et la Souris (Greenberg, Anderson et Tufrs, I936 ; Moore, Haliman et Sholi, ig38 ; Maynard et al., i958 ; Bunce, Jenkins et Phillips, i962 ; Hamuro, Shino et Suzuoki, I970). Depuis les observations initiales de Tufrs et GreEnbERG (I938), l'aggravation de la carence en magnésium par un régime riche en calcium ou phosphore a souvent été signalée. Le phosphore est nécessaire à l'apparition de la néphrocalcinose et accentue ses effets (ForBEs, I963). D'une façon générale, les calcifications aberrantes seraient plus sévères avec un régime riche en calcium ou phosphore (SEELIG, I97I; THOMAS et DURLACH, I97I) mais les effets de la seule surcharge du régime en calcium sont le plus souvent difficiles à interpréter car la teneur des régimes utilisés varie à la fois en phosphore et en calcium. Nous avons voulu tester l'influence de la teneur du régime en calcium sur l'évolution du déficit magnésique chez le Rat et sur l'apparition de néphrocalcinose. 


\section{MATÉRIEI ETT MÉTHODES}

Des rats Sherman de même sexe et de poids voisin sont répartis au hasard dans des groupes recevant des régimes différents. Les rats sont alimentés avec un régime semi-synthétique carencé en magnésium (30 p.p.m. estimé par dosage) ou avec un régime complémenté en cet élément (I 500 p.p.m.) par addition de $\mathrm{MgSO}_{4}$ (RAyssiguier, LaRvor et Barlet, I973). La teneur des régimes en calcium est de 6500 p.p.m. (régime normal en Ca), 500 p.p.m. (régime pauvre), I 4500 p.p.m. (régime riche), ces différentes teneurs étant obtenues en supprimant ou en ajoutant $\mathrm{CaCO}_{3}$ au régime de base. La teneur des régimes en phosphore ne varie pas (6 ioo p.p.m.). Les régimes suivants sont đonc utilisés : carencé en $\mathrm{Mg}$, normal en $\mathrm{Ca}: \mathrm{Mg}^{-} \mathrm{Ca}^{-}$; carencé en $\mathrm{Mg}$, pauvre en $\mathrm{Ca}: \mathrm{Mg}^{-} \mathrm{Ca}^{-}$; carencé en $\mathrm{Mg}$, riche en $\mathrm{Ca}: \mathrm{Mg}^{-} \mathrm{Ca}^{+}$; normal en $\mathrm{Mg}$, normal en $\mathrm{Ca}: \mathrm{Mg}^{=} \mathrm{Ca}^{-}$; normal en $\mathrm{Mg}$, pauvre en $\mathrm{Ca}: \mathrm{Mg}^{-} \mathrm{Ca}^{-}$; normal en $\mathrm{Mg}$, riche en $\mathrm{Ca}: \mathrm{Mg}^{=} \mathrm{Ca}^{+}$.

Avant l'abattage des animaux, le sang est récolté par ponction cardiaque dans une seringue héparinée ; il est immédiatement centrifugé puis selon les essais, le rein et le fémur gauches sont prélevés. Les dosages de Ca, Mg dans le plasma ou dans les tissus secs dégraissés par l'acétone et minéralisés à $55^{\circ} \mathrm{C}$ sont effectués par absorption atomique avec un appareil Perkin Elmer 303 selon les méthodes décrites par Perkin Elmer Corp. (I97I).

Les résultats sont comparés au moyen du test $t$ de Student. Dans les cas où les résultats ne suivent pas une distribution normale (calcifications rénales), les groupes ont été comparés selon un test non paramétrique de classement des données (SNEDECOR et COCHRAN, I967).

Trois essais successifs ont été réalisés selon les modalités suivantes:

\section{Essai 1}

Des rats mâles de 100 g environ sont répartis en 6 groupes de 8 animaux et reçoivent pendant I 4 jours les régimes précédemment décrits. L'alimentation est restreinte à $\mathbf{2} 2 \mathrm{~g} /$ jour.

\section{Essai 2}

Des rats mâles de $\mathrm{I} 30 \mathrm{~g}$ répartis en 4 groupes reçoivent les régimes $\mathrm{Mg}^{-} \mathrm{Ca}^{-} ; \mathrm{Mg}^{-} \mathrm{Ca}^{+}, \mathrm{Mg}^{-}$ $\mathrm{Ca}-\mathrm{Mg}^{-\mathrm{Ca}^{+}}$(alimentation ad libitum). Tous les jours pendant 8 jours, un lot de 5 rats est abattu dans chaque groupe. Un groupe supplémentaire de 5 rats est nourri avec un régime témoin $\mathrm{Mg}=\mathrm{Ca}^{-}$.

\section{Essai 3}

Des femelles de I Io g ( 98 animaux, nourris ad libitum) sont utilisées de la façon suivante : les rats répartis en 3 groupes (A, B et C) reçoivent pendant une période préliminaire de 7 jours un régime normal en $\mathrm{Mg}$ et à teneur en Ca normale ou surchargée. Puis ils reçoivent une ration carencée en $\mathrm{Mg}$ avec une teneur normale en $\mathrm{Ca}$ ou surchargée. La séquence des traitements était la suivante; groupe $\mathrm{A}: \mathrm{Mg}^{-} \mathrm{Ca}^{-}$puis $\mathrm{Mg}^{-} \mathrm{Ca}^{+}$; groupe $\mathrm{B}: \mathrm{Mg}^{-} \mathrm{Ca}^{+}$puis $\mathrm{Mg}^{-} \mathrm{Ca}^{+}$; groupe C : $\mathrm{Mg}^{=} \mathrm{Ca}=$ puis $\mathrm{Mg}^{-} \mathrm{Ca}=\mathrm{Au}$ cours de la $2^{\mathrm{e}}$ partie de l'expérience (carence en $\mathrm{Mg}$ ) 6 animaux étaient abattus chaque jour dans chaque groupe pendant ro jours. Le but de ce $3^{\text {e }}$ essai était de voir si une préparation préalable avec une ration surchargée en calcium pouvait prévenir les calcifications rénales plus efficacement que la surcharge calcique donnée seulement en même temps que la ration carencée en magnésium.

\section{RÉSULTATS}

\section{Essai 1}

Les signes caractéristiques de la carence en magnésium sont observés, en particulier la rougeur des oreilles, l'excitabilité accrue, l'appétit diminué et la perte de poids. Ces signes sont beaucoup plus précoces dans le groupe à ration riche en cal- 
TABLEAU I. - Essai no 1 : Influence de la teneur du régime en $\mathrm{Mg}$ et $\mathrm{Ca}$ sur la calcémie et la magnésémie du Rat après 2 semaines de traitement

(Ca et $\mathrm{Mg}: \mathrm{mg} / \mathrm{I}$ oo $\mathrm{ml}$; moyenne \pm erreur type $\sigma / \sqrt{\mathrm{N}}$; entre parenthèses, nombre d'animaux par groupe; les prélèvements de sang n'ayant pas été faits en même temps pour les groupes recevant un régime $\mathrm{Ca}^{-}$, les résultats de ces dosages ne sont pas mentionnés).

\section{First experiment : Effect of dietary $\mathrm{Mg}$ and $\mathrm{Ca}$ on calcemia} and magnesemia in rats after two weeks of treatment

(Ca and $\mathrm{Mg}: \mathrm{mg} / \mathrm{I} 00 \mathrm{ml}$; mean $\pm \mathrm{SE}$; between parentheses number of animals in each group). The significance of the difference between $\mathrm{Mg}^{-}, \mathrm{Mg}^{-}$is shown in the table. For the difference between $\mathrm{Ca}^{-} \mathrm{Ca}^{+}$, the significance is shown in the lowest part of the table.

\begin{tabular}{|c|c|c|c|c|}
\hline & & \multicolumn{2}{|c|}{$\begin{array}{c}\text { Dietary } M g \\
\text { Magnésium alimentaire }\end{array}$} & \multirow{2}{*}{$\begin{array}{l}\text { Signification de la } \\
\text { différence en fonction } \\
\text { de } \mathrm{Mg} \text { alimentaire }\end{array}$} \\
\hline & & $\mathrm{Mg}=1500$ p.p.m. & $\mathrm{Mg}^{-} 30$ p.p.m. & \\
\hline \multirow{2}{*}{$\begin{array}{l}\text { Dietary } \mathrm{Ca} \\
\text { Calcium } \\
\text { alimentaire }\end{array}$} & $\begin{array}{l}\mathrm{Ca}^{-} \\
500 \text { p.p.m. }\end{array}$ & $\begin{array}{c}\mathrm{Mg} 1,55 \pm 0,08 \\
(8) \\
\mathrm{Ca} 9,75 \pm 0,14\end{array}$ & $\begin{array}{c}\text { Mg } 0,29 \pm 0,03 \\
(8) \\
\text { Ca } 10,15 \pm 0,20\end{array}$ & $\begin{array}{c}\mathrm{P}<0,01 \\
\text { N. S. }\end{array}$ \\
\hline & $\begin{array}{c}\mathrm{C} a^{+} \\
14500 \text { p.p.m. }\end{array}$ & $\begin{array}{c}\mathrm{Mg} 1,13 \pm 0,03 \\
(8) \\
\text { Ca } 10,15 \pm 0,09\end{array}$ & $\begin{array}{c}\text { Mg } 0,18 \pm 0,01 \\
(7) \\
\text { Ca } 10,80 \pm 0,12\end{array}$ & $\begin{array}{l}P<0,01 \\
P<0,01\end{array}$ \\
\hline \multicolumn{2}{|c|}{$\begin{array}{l}\text { Signification de la différence } \\
\text { en fonction du } \mathrm{Ca} \text { alimentaire }\end{array}$} & $\begin{array}{l}\mathrm{Mg} \mathrm{P}<0,01 \\
\mathrm{Ca} \mathrm{P}<0,05\end{array}$ & $\begin{array}{l}\mathrm{Mg} \mathrm{P}<0,01 \\
\mathrm{Ca} P<0,05\end{array}$ & \\
\hline
\end{tabular}

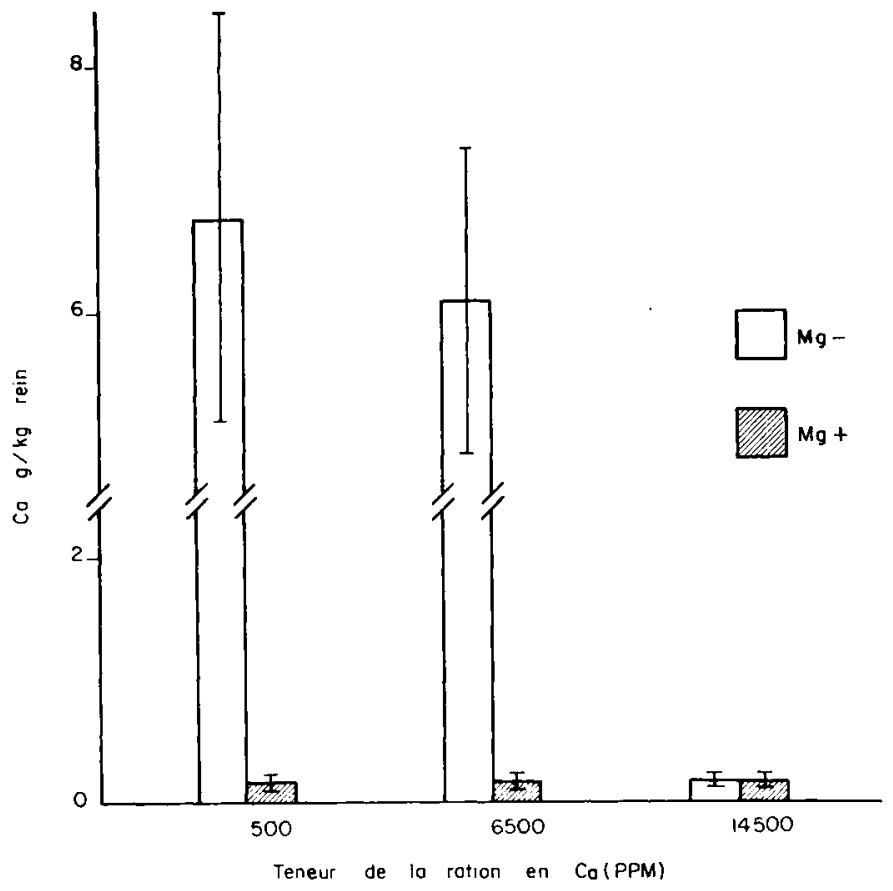

FIG. I. - Essai no 1 : Influence de la teneur du régime en Ca sur les calcifications rénales du rat après 2 semaines de déficit magnésique ( Ca $\mathrm{g} / \mathrm{kg}$ rein sec dégraissé $\left.\pm \frac{\sigma}{\sqrt{\mathrm{N}}}\right)$

Effect of dietary $\mathrm{Ca}$ on kidney calcifications in rat after two weeks of magnesium deficiency in the first experiment

(Ca $\mathrm{g} / \mathrm{kg}$ dry fat free kidney $\pm \mathrm{SE}$ ) 
TABLEAU 2

Essai no 2 : Influence de la teneur du régime en $M g$ et Ca sur la calcémie et la magnésémie du Rat après 8 jours de traitement

(même expression des résultats que pour le tableau I)

Second experiment : Effect of dietary $\mathrm{Mg}$ and $\mathrm{Ca}$ on calcemia and magnesemia in rat after 8 days of ireatment

(same expression of results than for table I)

\begin{tabular}{|c|c|c|c|c|}
\hline & & \multicolumn{2}{|c|}{$\begin{array}{c}\text { Dietary } M g \\
\text { Magnésium alimentaire }\end{array}$} & \multirow{2}{*}{$\begin{array}{c}\text { Signification de la } \\
\text { differrence en fonction } \\
\text { du Mg alimentaire }\end{array}$} \\
\hline & & $\mathrm{Mg}=1500$ p.p.m. & $\mathrm{Mg}^{-} 30$ p.p.m. & \\
\hline \multirow{2}{*}{$\begin{array}{l}\text { Dietary Ca } \\
\text { Calcium } \\
\text { alimentaire }\end{array}$} & $\begin{array}{c}\mathrm{Ca}^{-} \\
500 \text { p.p.m. }\end{array}$ & $\begin{array}{c}\operatorname{Mg} 1,40 \pm 0,02 \\
(5) \\
\text { Ca 11,33 } \pm 0,07\end{array}$ & $\begin{array}{c}\operatorname{Mg} 0,42 \pm 0,02 \\
(5) \\
\text { Ca } 10,67 \pm 0,13\end{array}$ & $\begin{array}{l}P<0,01 \\
P<0,01\end{array}$ \\
\hline & $\begin{array}{c}\mathrm{Ca}^{+} \\
14500 \text { p.p.m. }\end{array}$ & $\begin{array}{c}\operatorname{Mg} 1,28 \pm 0,13 \\
(5) \\
\operatorname{Ca} 11,93 \pm 0,24\end{array}$ & $\begin{array}{c}\mathrm{Mg} 0,33 \pm 0,02 \\
(5) \\
\mathrm{Ca} 12,55 \pm 0,24\end{array}$ & $\begin{array}{c}P<0,01 \\
\text { N. S. }\end{array}$ \\
\hline \multicolumn{2}{|c|}{$\begin{array}{l}\text { Signification de la différence } \\
\text { en fonction du } \mathrm{Ca} \text { alimentaire }\end{array}$} & $\begin{array}{c}\mathrm{Mg} \mathrm{N.} \mathrm{S.} \\
\mathrm{Ca} P<0,05\end{array}$ & $\begin{array}{l}\mathrm{Mg} \mathrm{P}<0,01 \\
\mathrm{Ca} \mathrm{P}<0,01\end{array}$ & \\
\hline
\end{tabular}

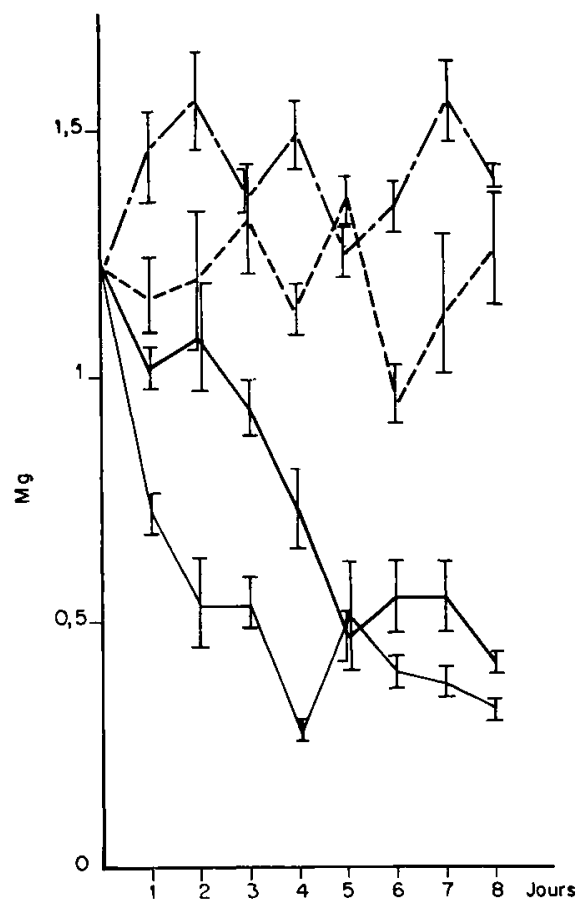

FIG. 2. - Évolution de la magnésémie (mg/100 $\mathrm{ml})$ en fonction des différents régimes utilisés dans l'essai $n^{\circ}$ z' Effect of dietary treatments on magnesemia in second experiment

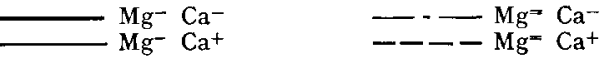



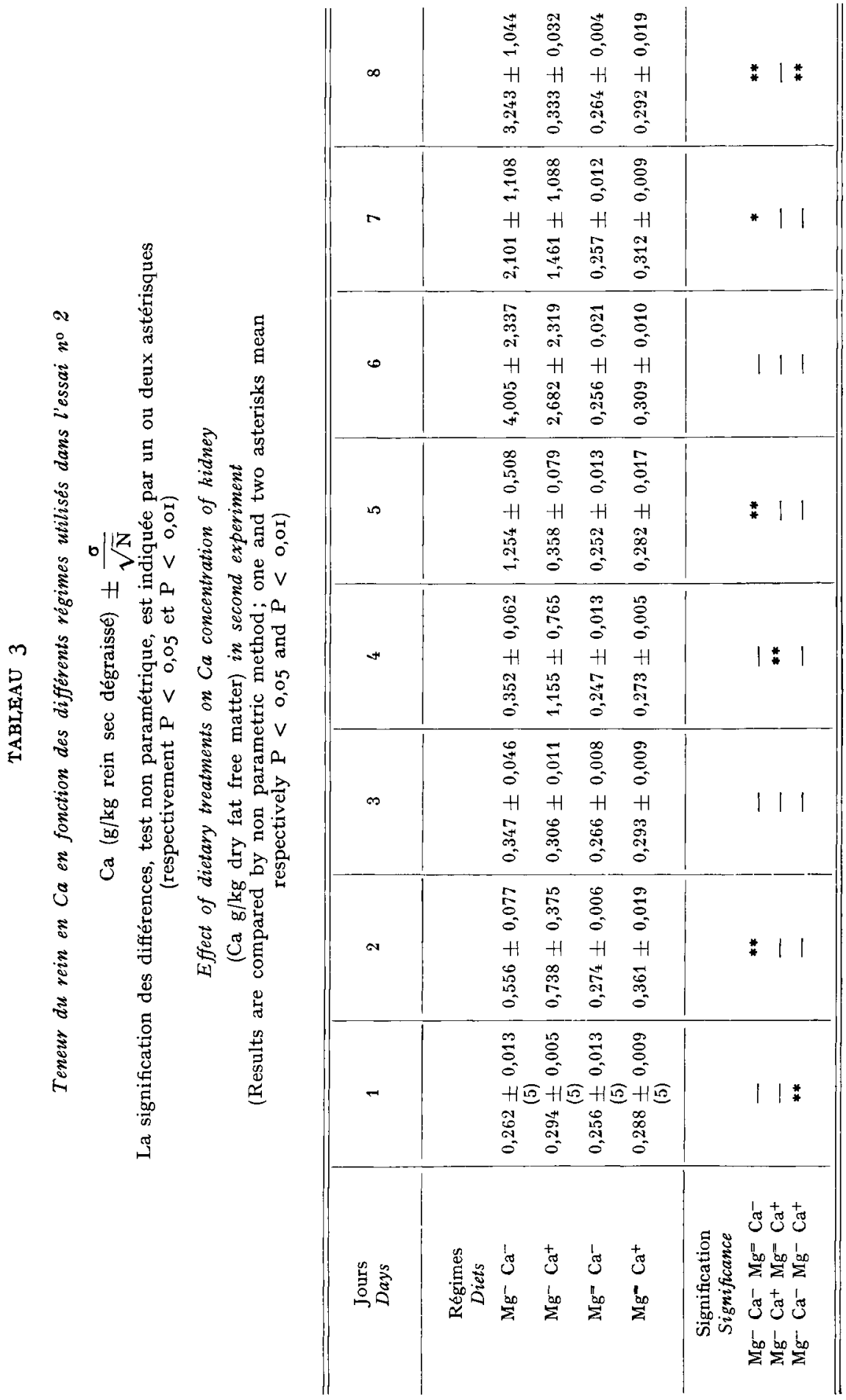


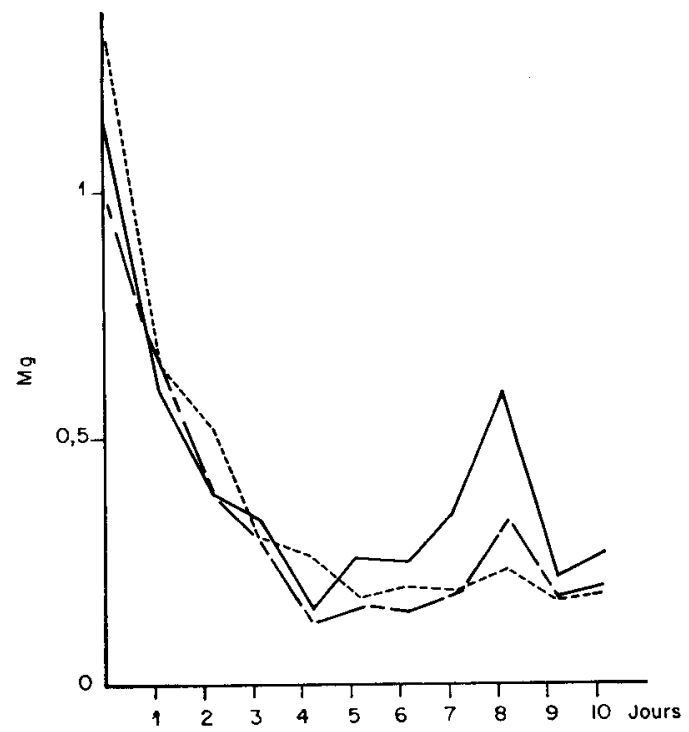

Fıg. 3. - Évolution de la magnésémie ( $\mathrm{mg} / \mathrm{too} \mathrm{ml}$ ) au cours de la carence en $\mathrm{Mg}$ en fonction des différents régimes utilisés dans l'essai no 3

Effect of dietary treatments on magnesemia in experiment 3

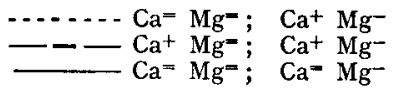

Teneur du rein en Ca en fonction des Groupe $A$ : $\mathrm{Mg}^{=} \mathrm{Ca}^{-}$; $\mathrm{Mg}^{-} \mathrm{Ca}^{+}$ Groupe $\mathrm{C}: \mathbf{M g}$

(Même expression des résu Effect of dietary treatments on $C d$ (Same expression o

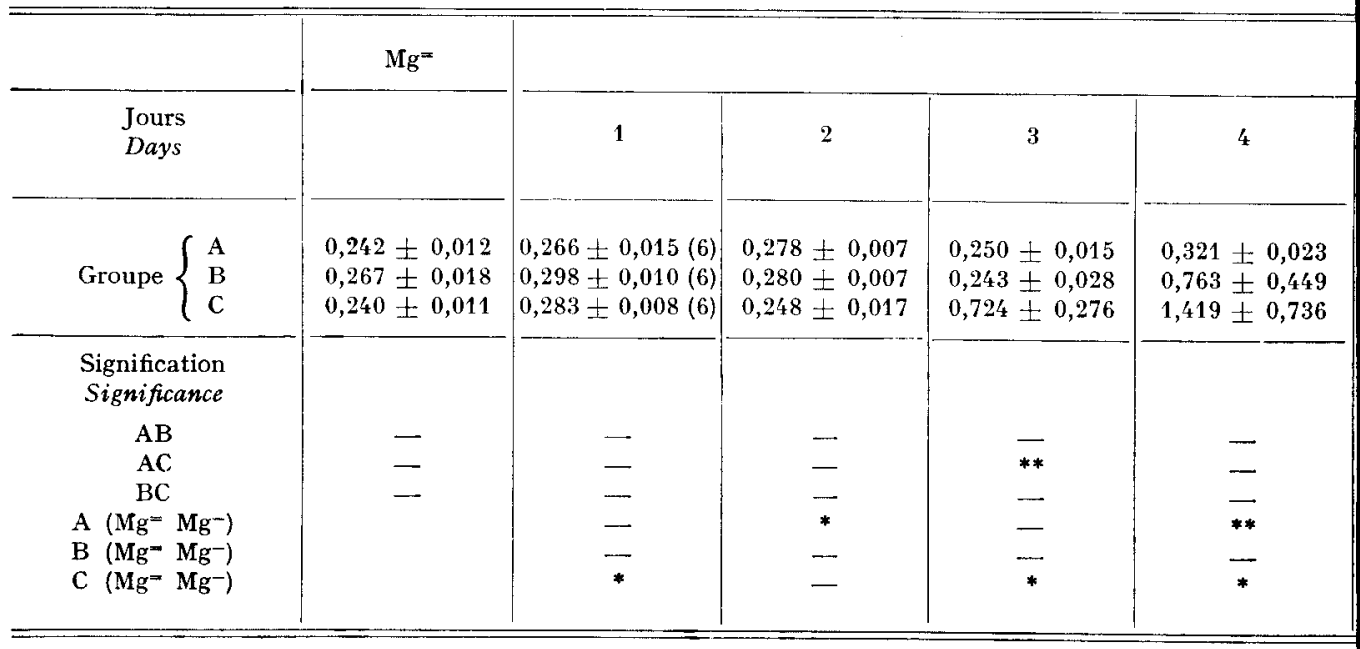


cium qui est le plus affecté par le déficit magnésique alors que la quantité de nourriture consommée est la même dans chaque groupe d'animaux. La carence en $\mathrm{Mg}$ s'accompagne d'hypomagnésémie et d'hypercalcémie mais la calcémie ne varie pas significativement chez les rats carencés à la fois en calcium et magnésium (tabl. I).

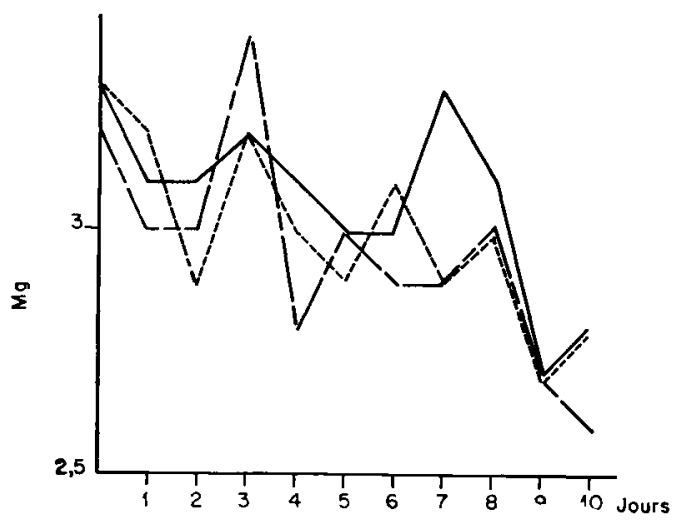

FIG. 4. - Évolution du magnésium osseux ( $\mathrm{Mg} \mathrm{g} / \mathrm{kg}$ os sec dégraissé) en fonction des différents régimes utilisés dans l'essai $n^{\circ} 3$

(même expression des résultats que pour la figure 3)

Effect of dietary treatments on $M g$ concentration of bone $(\mathrm{Mg} \mathrm{g} / \mathrm{kg}$ dry fat free matter) in experiment 3. (Same expression of results than for figure 3 )

EAU 4

diffévents régimes utilisés dans l'essai no 3

Groupe $\mathrm{B}: \mathrm{Mg}^{-} \mathrm{Ca}^{+} ; \mathrm{Mg}^{-} \mathrm{Ca}^{+}$;

$\mathrm{Ca}^{-} ; \mathrm{Mg}^{-} \mathrm{Ca}^{2}$

tats que pour le tableau 3)

concentration of kidney in experiment 3

results than for table 3 )

\begin{tabular}{|c|c|c|c|c|c|}
\hline \multicolumn{6}{|c|}{$\mathrm{Mg}^{-}$} \\
\hline 5 & 6 & 7 & 8 & 9 & 10 \\
\hline $0,399 \pm 0,088$ & $0,329 \pm 0,037$ & $0,583 \pm 0,240$ & $1,013 \pm 0,322$ & $0,373 \pm 0,056$ & $1,520 \pm 0,778$ \\
\hline $0,684 \pm 0,279$ & $0,616 \pm 0,319$ & $1,648 \pm 0,885$ & $2,430 \pm 0,912$ & $0,713 \pm 0,318$ & $0,956 \pm 0,257$ \\
\hline $2,296 \pm 0,861$ & $8,160 \pm 3,937$ & $2,949 \pm 0,854$ & $5,430 \pm 1,306$ & 5,748 七 2,158 & $2,728 \pm 1,108$ \\
\hline 一 & 一 & 一 & - & $\ldots$ & - \\
\hline - & $* *$ & 一 & * & ** & - \\
\hline- & ** & - & - & ** & $\ldots$ \\
\hline$*$ & * & ** & ** & ** & * \\
\hline - & 一 & - & $* *$ & $*$ & ** \\
\hline - & $* *$ & $* *$ & $* *$ & ** & $* *$ \\
\hline
\end{tabular}


La magnésémie est diminuée par la charge du régime en calcium quelle que soit la teneur de la ration en magnésium. Un régime riche en calcium permet de prévenir totalement l'apparition de calcifications rénales qui sont particulièrement intenses avec un régime carencé en calcium et en magnésium (fig. I).

\section{Essai 2}

La magnésémie et la calcémie des animaux après 8 jours de carence sont données dans le tableau 2. La figure 2 et le tableau 3 indiquent l'évolution journalière de la magnésémie et du calcium rénal. Les rats carencés à la fois en $\mathrm{Ca}$ et $\mathrm{Mg}$ deviennent hypocalcémiques par rapport aux animaux déficients uniquement en $\mathrm{Ca}(\mathrm{P}<0, \mathrm{OI})$. Des calcifications rénales apparaissent de façon variable chez les rats déficients en $\mathrm{Mg}$ au début de la carence quelle que soit la teneur du régime en $\mathrm{Ca}$ et disparaissent $(\mathrm{P}<\mathrm{o}, \mathrm{OI})$ après 8 jours d'un régime riche en $\mathrm{Ca}$.

\section{Essai 3}

Les figures 3 et 4 et le tableau 4 représentent les variations de la magnésémie, du Mg fémoral et des calcifications rénales avec les différents régimes utilisés. Dans le cas d'un régime riche en $\mathrm{Ca}$, les calcifications rénales du déficit magnésique sont moins importantes qu'avec une ration pauvre en $\mathrm{Ca}$ mais subsistent après ro jours de carence. Il n'y a pas de différence significative dans la teneur du rein en $\mathrm{Ca}$, que la ration ait été ou non enrichie en $\mathrm{Ca}$ avant le début du déficit magnésique. Le $8 \mathrm{e}$ jour on observe une aggravation de la néphrocalcinose et une augmentation de la magnésémie, mais il n'y a pas de corrélation significative entre la magnésémie et la teneur du rein en calcium.

\section{DISCUSSION}

L'hypomagnésémie, l'hypercalcémie, les calcifications rénales sont des données classiques du déficit magnésique chez le Rat. Nous avons vu que la magnésémie a tendance à diminuer chez les animaux surchargés en $\mathrm{Ca}$, carencés ou non en magnésium. L'aggravation du déficit magnésique par une surcharge calcique peut être rapportée à plusieurs phénomènes. La baisse de la digestibilité $\mathrm{du} \mathrm{Mg}$ a souvent été observée, et ne semble pas pouvoir être attribuée à une compétition entre l'absorption intestinale de $\mathrm{Ca}$ et $\mathrm{Mg}$. Les travaux réalisés in vitro ne sont pas toujours très concluants (SCHACHTER et ROSEN, I959). Lors du déficit magnésique, l'absorption intestinale de magnésium est de toute façon très limitée en raison de la faible teneur du régime en cet élément et dans ces conditions l'excès de calcium ne peut guère diminuer 1'absorption du magnésium, bien qu'on ne puisse exclure une augmentation de l'excrétion fécale endogène du Mg. Une autre possibilité est celle d'une mobilisation des réserves osseuses plus difficile par baisse de la sécrétion de la parathormone mais l'évolution de la composition osseuse (fig. 4) ne permet pas d'appuyer une telle hypothèse. Nous n'avons pas réalisé de bilan mais les pertes urinaires en $\mathrm{Mg}$ augmentent d'une façon générale si l'on administre du calcium (WOLF et BALI, I949; 
BARKER, EIKINTON et ClaRK, I959) et peuvent intervenir pour expliquer la baisse de la magnésémie.

Si les rats sont carencés à la fois en $\mathrm{Ca}$ et $\mathrm{Mg}$, l'hypercalcémie fait place à une hypocalcémie (tabl. 2) en accord avec les résultats de HEATON et ANDERSON (1965) et de Mac Manus et HEATon (I969). La tendance à l'hypercalcémie observée dans le premier essai pourrait être rapprochée des résultats inexpliqués de RichaRDson et WELT (I963) qui indiquent une hypercalcémie chez des rats déficients à la fois en $\mathrm{Ca}$ et $\mathrm{Mg}$. L'hypercalcémie au cours de la carence en Mg paraît donc résulter principalement chez le Rat d'une augmentation de l'assimilation intestinale de $\mathrm{Ca}$, bien qu'on ne puisse exclure totalement la participation d'une modification métabolique non définie.

Un régime pauvre en $\mathrm{Ca}$ n'inhibe pas les calcifications rénales, contrairement aux résultats de HEATON et ANDERSON (I965) chez des animaux, il est vrai, à la magnésémie assez élevée. Le calcium de la ration n'est pas indispensable à l'apparition de calcifications rénales qui disparaissent au contraire avec une ration riche en cet élément. Chez le Rat, la présence de parathyroïdes fonctionnelles conditionnent l'apparition de néphrocalcinose (HEATON et ANDERSON, I965) qui peut être supprimée par l'injection de thyrocalcitonine (FARNELL et WHITEHAIR, I97I). Le régime riche en $\mathrm{Ca}$ administré dans nos essais est susceptible d'inhiber la sécrétion de parathormone ou de stimuler les cellules $\mathrm{C}$ de la thyroïde mais il est difficile de savoir si la disparition des calcifications rénales est liée à 1'un ou 1'autre de ces phénomènes. Avec ce régime les calcifications du début de la carence en $\mathrm{Mg}$ sont plus ou moins longues à disparaître mais l'administration d'un régime enrichi en calcium une semaine avant le début du déficit magnésique ne modifie pas la valeur du Ca rénal par rapport au taux observé chez des animaux ayant une alimentation calcique normale avant le début de la carence. Certaines calcifications peuvent donc apparaître après une administration prolongée d'un régime susceptible d'entraîner une large inhibition des parathyroïdes. L'hypothèse d'un hyperparathyroïdisme responsable de la néphrocalcinose pourrait dans ces conditions être écartée. L'hypercalcémie de la carence subsiste avec l'administration d'un régime riche en $\mathrm{Ca}$ et un hyperparathyroïdisme éventuel ne peut donc également expliquer l'hypercalcémie du déficit magnésique qui serait liée chez le Rat à une résorption intestinale accrue du Ca alimentaire (LARVOR et DURLACH, I97I). Hypercalcémie et néphrocalcinose sont deux phénomènes distincts, les calcifications aberrantes intervenant beaucoup plus précocement $(48 \mathrm{~h})$ que l'hypercalcémie et pouvant s'accompagner d'hypocalcémie dans le cas d'un régime pauvre en Ca.

Le problème de la sécrétion parathyroïdienne n'est pas parfaitement élucidé en l'absence c̀e dosage radioimmunologique de la P'TH chez le Rat carencé en Mg mais un hyperparathyroïdisme lors du déficit magnésique semble peu probable en dépit de l'hypercalcémie, de 1'hypophosphatémie et des calcifications aberrantes (LARVOR et DURIACH, I97I). Le déficit magnésique pourrait s'accompagner dans certaines espèces d'un hypoparathyroïdisme (ANAST et al., I972).

En ce qui concerne l'influence d'une sécrétion accrue de calcitonine avec notre régime riche en calcium, il faut remarquer que de toute façon la carence en $\mathrm{Mg}$ s'accompagne chez le Rat d'une hypertrophie et d'une hyperplasie des cellules C (JASmin, I968; Stachura et PFarse, I970).

Une autre possibilité pour expliquer l'inhibition des calcifications par un régime 
riche en Ca doit être envisagée. La néphrocalcinose induite chez le Mouton par un régime riche en phosphore peut être améliorée par une surcharge alimentaire en Ca qui réduit l'élimination urinaire de phosphore (Bushman, EMERICK et FMBRY, I965).

La carence en $\mathrm{Mg}$ s'accompagne d'hypocalciurie et d'hyperphosphaturie même chez les animaux parathyroïdectomisés (GINN et SHANBour, I967). L'addition de Ca, en réduisant l'élimination du phosphore et en entraînant une hypercalciurie, pourrait rendre plus difficile les phénomènes de calcifications rénales par une modification du rapport $\mathrm{Ca} / \mathrm{P}$ urinaire. ForBEs (I963) note cependant que de telles calcifications peuvent intervenir avec des taux de $\mathrm{Ca} / \mathrm{P}$ urinaires assez variables.

La néphrocalcinose ne constitue qu'un aspect particulier de troubles plus généraux du métabolisme calcique chez l'animal carencé en magnésium. Les calcifications elles-mêmes pourraient, par les lésions provoquées, aggraver de telles perturbations puisque l'on connaît d'une part la baisse de la réceptivité à la vitamine $D$ chez le Rat carencé en Mg (LIFSHITZ, HARRISON et HARRISON, I967) et d'autre part le rôle du rein dans la formation du I-25-dihydroxycholécalciférol. La lithiase phosphatique du déficit magnésique peut être inhibée par des traitements divers ou la mise en jeu de modifications endocriniennes ou alimentaires et nous montrons dans ces essais le rôle prophylactique d'une ration enrichie en calcium.

En dépit de nombreuses tentatives, le mécanisme responsable de la néphrocalcinose est inconnu en raison très certainement d'une connaissance insuffisante des modifications endocriniennes du déficit magnésique.

Reçu pour publication en juin 1973.

\section{SUMMARY}

\section{EFFECT OF DIETARY CALCIUM ON KIDNEY CALCIFICATION, MAGNESEMIA} AND BONE MAGNESIUM OF MAGNESIUM DEFICIENT RATS

A high dietary Ca enhances symptoms of magnesium deficiency and it has been suggested that renal and cardiovascular calcinosis induced in magnesium deficient animals might be aggravated by $\mathrm{Ca}$ supplementation. The present experiments were conducted in order to investigate this possibility.

Sherman rats of same sex and weight in each experiment were randomly divided into the required number of groups and received appropriate diets. Composition of the low magnesium diet $\left(\mathrm{Mg}^{-}\right.$30 p.p.m. $\mathrm{Mg}$ ) and the magnesium supplemented $\operatorname{diet}\left(\mathrm{Mg}^{=}{ }_{\mathrm{I}}\right.$ 500 p.p.m. $\left.\mathrm{Mg}\right)$ has been published elsewhere. Calcium content was adjusted to required levels $\left(\mathrm{Ca}^{-} 500, \mathrm{Ca}^{-} 6500\right.$, $\mathrm{Ca}^{+} \mathrm{r}_{4} 500$ p.p.m.) by varying the level of $\mathrm{CaCO}_{3}$. Magnesium and calcium were estimated in plasma and kidney by atomic absorption spectrophotometry.

In the first experiment, rats $\left(\delta^{*}\right)$ were divided into 6 groups of 8 animals receiving the following diets : $\mathrm{Mg}^{-} \mathrm{Ca}^{-} ; \mathrm{Mg}^{-} \mathrm{Ca}^{-} ; \mathrm{Mg}^{-} \mathrm{Ca}^{+} ; \mathrm{Mg}^{-} \mathrm{Ca}^{-} ; \mathrm{Mg}^{-} \mathrm{Ca}^{-} ; \mathrm{Mg}^{-} \mathrm{Ca}^{+} ;$during two weeks. In the $\mathrm{Mg}^{-} \mathrm{Ca}^{+}$groups compared to the $\mathrm{Mg}^{-} \mathrm{Ca}^{-}$group, clinical signs of deficiency (hyperaemia of the ears, hyperexcitability) appeared earlier; plasma $\mathrm{Mg}$ concentration was lower (table I). but there was inhibition of kidney calcification with increase in dietary calcium, the resulting level of kidney calcium being no different from controls $\mathrm{Mg}^{=\mathrm{Ca}^{+}}$(fig. I).

In the second experiment with $\mathrm{Mg}^{-} \mathrm{Ca}^{-} ; \mathrm{Mg}^{-} \mathrm{Ca}^{+} ; \mathrm{Mg}^{=\mathrm{Ca}^{-}} ; \mathrm{Mg}^{=} \mathrm{Ca}^{+}$diets, five rats $\left(\sigma^{+}\right)$ were killed during 8 days in each group. Plasma Mg concentration was reduced in Mg deficient groups and hypercalcemia was observed in $\mathrm{Mg}^{-} \mathrm{Ca}^{+}$compared with $\mathrm{Mg}^{-} \mathrm{Ca}^{+}$, but plasma $\mathrm{Ca}$ concentration was lowered in $\mathrm{Mg}^{-} \mathrm{Ca}^{-}$compared with $\mathrm{Mg}^{-} \mathrm{Ca}^{-}$(fig. 2, table 2). Classical hypercalcemia of magnesium deficiency in rat requires the presence of calcium in the diet. Calcification of the kidney was initiated very early in $\mathrm{Mg}$ deficiency, whatever the calcium level of the diet was, but disappeared after a week of $\mathrm{Mg}^{-} \mathrm{Ca}^{+}$diet (table 3 ). 
Experiment 3 was destined to investigate effects of feeding a high calcium level diet to rats (O) before magnesium deficiency. Group A was fed $\mathrm{Mg}^{-} \mathrm{Ca}^{=}$for 7 days, then $\mathrm{Mg}^{-} \mathrm{Ca}+$ for $\mathrm{Io}$ days ; group $\mathrm{B}$ received the $\mathrm{Mg}=\mathrm{Ca},{ }^{+} \mathrm{Mg}-\mathrm{Ca}^{+}$diets ; and group $\mathrm{C}, \mathrm{Mg}=\mathrm{Ca}=, \mathrm{Mg}^{-} \mathrm{Ca}^{-}$diets. Six rats of each group were killed daily during the magnesium deficiency. Effects of dietary treatment on plasma $\mathrm{Mg}$ concentration and bone $\mathrm{Mg}$ concentration are shown in figure 3 and 4 . Early calcification of the kidney did not disappear in group $B$ and was not significantly different from group A (table 4 ).

From our data, it is difficult to suggest the nature of preventive action of calcium on renal calcinosis. Parathyroid activity can be depressed by high dietary calcium and, in rat, removal of the parathyroid gland prevents any rise in kidney calcium concentration (HEATON and ANDERsoN, I 965). However, hyperparathyroidism has not been established during magnesium deficiency in rats, and it seems doubtful that hyperactivity of the parathyroid glands during $\mathrm{Mg}$ deficiency induces kidney calcification. In our experiments, when rats were fed extra calcium before magne. sium deficiency, early calcification did not disappear with a lower parathyroid activity. TCT can prevent renal calcification (FARNELL and WHITEHAIR, I97I), but Mg deficiency in rat seems associated with increased calcitonin secretion. Experimentally induced ovine phosphatic urolithiasis can be reduced by raising the level of dietary calcium, which lowers urine phosphate level (Bushman, EMERICK and EMBRY, 1965). Mg deficiency has been shown to induce an increased P urinary excretion (in normal or PTX rats) and a decreased urinary excretion of Ca. Addition of $\mathrm{Ca}$ decreased $\mathbf{P}$ urinary excretion, and increased Ca excretion. Modification of the Ca/P ratio in the urine could prevent kidney calcification.

\section{RÉFÉRENCES BIBLIOGRAPHIQUES}

ANonyme, 1971. Analytical methods for atomic absorption spectrophotometry. The Perkin Elmer Corp. Publisher, Norwalk Conn.

Anast C. S., Mohs J. M., Kaplan S. L., Burns T. W., 1972. Evidence for parathyroid failure in magnesium deficiency. Science, 177, 606-608.

Barker E. S., Elkinton J. R., Clark J. K., 1959. Studies on renal excretion of magnesium in Man J. Clin. Invest., 38, I733-I745.

Bunce G. E., Jenkins K. J., Phillips P. H., r962. The mineral requirement of the dog. 3. The magnesium requirement. $J$. Nutr., 76, I7-22.

Bushman D. H., Emerick R. J., Embry L. B., 1965. Experimentally induced ovine phosphatic urolithiasis. Relationships involving dietary calcium, phosphorus and magnesium. J. Nutr., 87, 499504 .

FarNell D. R., Whitehair C. K., 197I. Influence of thyrocalcitonin in rats fed magnesium deficient and complete rations. Am. J. Vet. Res., 32, $132-148$.

Forbes R. M., I963. Mineral utilisation in the Rat. I. Effect of varying dietary ratios of calcium, magnesium and phosphorus. J. Nutr., 80, 322-326.

Ginn H. E., Shanbour L. L., 1967. Phosphaturia in magnesium deficient rats. Amer. J. Physiol., 212, I 347-I 350 .

Greenberg D. M., Anderson C. E., Tufts E. V., 1936. Pathological changes in tissues of rats reared on diets low in magnesium. J. Biol. Chem., 114, 43.

Hamuro Y., Shino A., Suzuoki Z., ig7o. Acute induction of soft tissue calcification with transient hyperphosphatemia in the $\mathrm{KK}$ mouse by modification in dietary contents of calcium, phosphorus and magnesium. J. Nutr., 100, 404-412.

Heaton F. W., Anderson C. K., 1965. The mechanism of renal calcification induced by magnesium deficiency in the rat. Clin. Sci, 28, 99-106.

Jasmin G., 1968. Action of hormones on the progression of magnesium deficiency syndrome in rats. In : Endocrine aspects of disease process. 356-387. Warren H., Green Inc. Publisher, Saint-Louis, Missouri.

LaRvor P., DURLach J., I97I. Rapport physiologique entre magnésium et glandes endocrines. In : First International Symposium on magnesium deficit in human pathology, 25I-296. J. Durlach, édit., Vittel.

Lifshitz F., Harrison H. C., Harrison H. E., r967. Response to vitamin D of magnesium deficient rats. Proc. Soc. Exptl. Med., 125, 472-476.

Mac Manus J., Heaton F. W., 1969. The effect of magnesium deficiency on calcium homeostasis in the rat. Clin. Sci., 36, 296-306.

Maynard L. A., Boggs D., Fisk G., Seguin D., 1958. Dietary mineral interrelations as a cause of soft tissue calcification in guinea pigs. J. Nutr., 64, 85-97.

Moore L. A., Hallman E. T., Shol, L. B., I938. Cardiovascular and other lesions in calves fed diets low in magnesium. Arch. Pathol., 26, 820-838. 
Rayssiguier Y., Larvor P., Barlet J. P., i973. Étude de l'influence d'un phosphonate de sodium sur le taux rénal de calcium chez le rat carencé en magnésium. C. R. A cad. Sci., 276, série D, 2035-2038.

Richardson J. A., Welt L. G., r963. The hypercalcemia of magnesium depletion. J. Clin. Invest., 42, $970-97 \mathrm{I}$.

Schachter D., Rosen S. M., 1959. Active transport of ${ }^{45} \mathrm{Ca}$ by the small intestine and its dependance on vitamin D. Amer. J. Physiol., 196, 357-362.

SEELIG M. S., I97I. Human requirement of magnesium factors that increase needs. In : First International Symposium on Magnesium deficit in human pathology. II-38. J. Durlach, edit. Vittel.

SNedecor G. W., Cochran W. G., I967. Non parametric methods. In : Statistical methods, r30-131. $6^{\mathrm{e}}$ édition. Iowa State University press, Ames Iowa U. S. A.

Stachura J., Pearse A. G. E., I97o. Thyroid C cells in experimental hyper and hypomagnesaemia. Virchows Arch. Abt. B. Zellpath., 5, I73-186.

Tномas J., Durlach J., 197r. Déficit magnésique et pathologie du rein. In : First International Symposium on magnesium deficit in human pathology. 359-385. J. Durlach, edit. Vittel.

TuFts E. V., Greenberg D. M., 1938. Biochemistry of magnesium deficiency. Minimum magnesium required for growth, gestation and lactation and effect of dietary calcium level thereon. J. Biol. Chem., $122,715-726$.

Wolf A. V., Ball S. M., 1949. Effect of intravenous calcium salts on renal excretion in the dog. Amer. J. Physiol., 158, 205-217. 